\title{
Utility of 18S rDNA and ITS sequences as population markers for Lepeophtheirus salmonis (Copepoda: Caligidae) parasitising Atlantic salmon (Salmo salar) in Scotland
}

\author{
${ }^{1}$ A.P. Shinn, ${ }^{1}$ B.A. Banks, ${ }^{2}$ N. Tange, ${ }^{1}$ J.E. Bron, ${ }^{1}$ C. Sommerville, ${ }^{2}$ T. Aoki \& ${ }^{1}$ R.Wootten \\ Institute of Aquaculture, University of Stirling, Stirling FK9 4LA, Scotland; '2Laboratory of Genetics and \\ Biochemistry, Department of Aquatic Bioscience, Tokyo University of Fisheries, Konan 4-5-7, Minato-ku, \\ Tokyo 108, Japan
}

Keywords: Sea louse, Lepeophtheirus salmonis, copepod, Crustacea, Atlantic salmon, sequencing, 18S ribosomal DNA, internal transcribed spacer, population genetics

\begin{abstract}
Genetic differentiation within the salmon louse Lepeophtheirus salmonis (Krøyer, 1837), was investigated by the sequencing of specific nucleotide regions. Partial sequences of the $18 \mathrm{~S}$ ribosomal RNA gene and the ribosomal internal transcribed spacer (ITS-1) region from single sea lice were amplified by the polymerase chain reaction (PCR). Lice were collected from wild and farmed Atlantic salmon (Salmo salar L., 1758) from nine selected localities around the Scottish coastline. A $0.9 \mathrm{~kb}$ fragment of the 18S ribosomal RNA gene was amplified and compared for several samples of lice which showed no observable differences between the lice from different collection sites confirming the absence of cryptic species. The 454 nucleotide ITS-1 sequence showed differences between derived sequences from 13 sea lice samples from 4 collection sites which included 2 farm sites and 2 sites where lice were taken from wild fish. Across all samples, there was a 92.14\% similarity in the ITS-1 sequence. The percentage similarity in the ITS-1 sequence in samples of lice from two fish farms were $99.71 \%$ (site A) and $95.72 \%$ (site D) but only $86.90 \%$ (site B) and $86.03 \%$ (site C) simîlarity was shown in lice samples taken from sites where wild salmonids were caught. The greater similarity between the ITS-1 sequence within farm sites may be attributed to a restricted gene flow within lice populations in Atlantic salmon cage sites.
\end{abstract}

\section{Contents}

Introduction $\quad 89$

Material and methods $\quad 90$

Sample collection and preparation $\quad 90$

Genomic DNA extraction 91

PCR amplification $\quad 91$

18S ribosomal DNA $\quad 91$

Internal Transcribed Spacer (ITS) 91

Cloning ínto pMOS Blue T-vector 91

Dideoxynucleotide sequencing and analysis $\quad 92$ $\begin{array}{ll}\text { Results } & 92 \\ 18 S \text { ribosomal DNA } & 92 \\ \text { Internal Transcribed Spacer (ITS-I) } & 92 \\ \text { Discussion } & 93 \\ \text { Acknowledgements } & 97 \\ \text { References } & 97\end{array}$

\section{Introduction}

The characterization of salmon louse (Lepeophtheirus salmonis (Krøyer, 1837)) populations has recently become the subject of considerable interest as a tool to investigate louse population origins and interactions. Nordhagen, Heuch \& Schram (1998) investigated experimentally the usefulness of morphometric parameters to identify the origins of the salmon lice populations in Norway. They concluded that it was not possible to discriminate populations of lice on the basis of size, as lice of different origins raised at the same temperature on a common host showed no significant differences in dimensions and similar life-cycle features such as egg production and growth rate. Similarly, in an unpublished experiment, the present authors applying multivariate analyses to morphometric data of several populations of lice taken from naturally infected wild and farmed salmonids sampled throughout a year, concluded that the discrimination of lice on the basis of size was not sufficiently clear to be useful.

. Other studies have investigated the allelic frequencies of polymorphic enzymes (allozymes) of populations of the genus Lepeophtheirus. For example, Shinn et al. (1998) found that, using nine 
polymorphíc enzymes, it was possible to obtain some discrimination of lice from wild fish on the east coast of Scotland from both wild and farmed fish sampled on the west coast of Scotland. Isdal, Nylund \& Nævdal's (1997) allozyme study of Norwegian populations of lice similarly found differences between northern and southern populations of farmed lice. De Meeüs et al. (1992) used allozymes to demonstrate an adaptive polymorphism to salinity in L. europaensis Zeddam, Berrebi, Renaud, Raibaut \& Gabrion, 1988 parasitic on Scophthalmus rhombus L., 1758 and Platichthys flesus L., 1758 which they suggested, when coupled with a restricted movement of hosts between environments of differing salinity, could in time give rise to genetically isolated sub-populations.

Other approaches in the investigation of the population characteristics of $L$. salmonis include the use of inductively coupled plasma mass spectrometry (ICPMS) to ascertain the elemental signature of adult female lice (Shinn et al., this volume). In this study it was demonstrated that adult female lice from seven localities could be discriminated from each other on the basis of 16 elements and lice from farmed fish could be discriminated from lice on wild salmonids within the same localíty.

Molecular techniques permit finer resolution of genetic differences between populations and individuals that cannot be detected using techniques such as morphometrics and allozyme analysis. Todd et al. (1997) using RAPDs, stated that genetic differentiation between populations of lice on farmed salmon and wild salmonids and between individual farm sites was evident to the extent that the presence of putative "farm markers" within certain individual wild lice suggested they originated from farm sites. While RAPDs allow for the comparison of amplified DNA bands of similar molecular weight, without direct sampling it is unknown whether these similar sized bands correspond to the same portion of the genome. This study describes an investigation which characterizes populations through determination of the nucleotide sequence of selected areas of the $L$. salmonis genome, namely the $18 \mathrm{~S}$ ribosomal RNA gene and the internal transcribed spacer (ITS).

Information resulting from analysis of the $18 \mathrm{~S}$
rRNA gene sequence has previously been used to determine whether or not the species under investigation contains cryptic species or genetically isolated populations. Similarly, determination of the nucleotide sequence of internal transcribed spacers (ITS) have been successfully implemented as a taxonomic tool to discriminate closely related or morphologically indistinguishable species of parasite e.g apicomplexans (Homan et al., 1997); gyrodactylids (Monogenea) (Cunningham, McGillivray \& MacKenzie, 1995; Cunningham, 1997); schistosomes (Digenea) (Littlewood \& Johnston, 1995; Van Herwerden, Blair \& Agatsuma, 1998); and nematodes (Chilton, Gasser \& Beveridge, 1995). Nucleotide variations in ITS regions have also been used to characterise strains or isolates of Cyptocaryon irritans (Diggles \& Adlard, 1997); Enterocytozoon bieneusi (Rinder, KatzwinkelWladarsch \& Loscher, 1997a); Eimeria maxima (Barta et al., 1998); Echinostoma caproni (Morgan \& Blair, 1998) and Hymenolepis diminuta (Okamoto et al., 1997). Sequence similarity in the ITS region has also provided evidence for synonomy eg. Teladorsagia spp. (Trichostrongylidae) (Stevenson, Gasser \& Chilton, 1996) and Fasciola spp. (Hashimoto et al., 1997); sub-speciation e.g. Echinococcus multilocularis (Rinder et al., 1997b) and Paragonimus westermani (Blair et al., 1997) or the identification of cryptic species as has been suggested for geographically distanced isolates of Necator americanus (Romstad et al., 1998). Overall, these studies suggest that the $18 \mathrm{~S}$ and ITS regions may be used to provide a means of population characterization and it is with this in mind that this study has been undertaken.

\section{Materials and methods}

\section{Sample collection and preparation}

Sea lice were collected from eight sites in Scotland. Samples were collected from wild Atlantic salmon (Salmo salar L., 1758) at three sites and from farmed Atlantic salmon at five sites (Fig. 1). After collection sea lice were maintained in sea water $\left(10^{\circ} \mathrm{C}\right)$ for $48 \mathrm{hrs}$ to remove host products from the gut. before use and then homogenised in 
buffer (1mM Tris-HCl pH7.0, EDTA $1 \mathrm{mM}, 2-$ mercaptoethanol $0.1 \mathrm{mM}$ ). Samples were then frozen in liquid nitrogen and stored at $-70^{\circ} \mathrm{C}$.

\section{Genomic DNA extraction}

Frozen lice samples were kept on ice, homogenised in a cell lysis buffer ( $10 \mathrm{mM}$ Tris- $\mathrm{HCl} \mathrm{pH} 8.0$; $150 \mathrm{mM} \mathrm{NaCl} ; 10 \mathrm{mM}$ EDTA-NaOH pH 8.0; and $1 \% \mathrm{SDS})$ and then treated with proteinase $\mathrm{K}(1 \mathrm{mg} /$ $\mathrm{ml})$ overnight at $55^{\circ} \mathrm{C}$ and RNase $\mathrm{A}(0.5 \mathrm{mg} / \mathrm{ml})$ for one hour at $37^{\circ} \mathrm{C}$ to digest cellular proteins and RNA. Genomic DNA was then precipitated using $0.3 \mathrm{M} \mathrm{NaOH}-\mathrm{Ac}$ and isolated using a standard phenolic-chloroform extraction (Sambrook et al., 1989) and precipitated in ice-cold $75 \%$ ethanol. The purified DNA pellets were dried at room temperature overnight and resuspended in TE buffer (10mM Tris- $\mathrm{HCl} \mathrm{pH} 8.0,1 \mathrm{mM}$ EDTA).

\section{PCR amplification}

Universal and species specific primers (given below) were selected for each gene and amplified using PCR (Saiki et al., 1988).

\section{$18 S$ ribosomal DNA}

The nematode Caenorhabditis elegans rRNA primers complementary to the annealing positions 20742098 5'-GGGCAAGTCTGGTGCC-3' (18S-1) and 2931-2946 5'-GGTCTGTGATGCCCTT-3' (18S2) were used (Ellis, Sulston \& Coulson, 1986). A $25 \mu \mathrm{l}$ reaction mix was used, containing $25 \mathrm{pM}$ of each primer, 50-100ng DNA and a PCR bead (Amersham Pharmacia-Biotech)(1.5 units of Taq, $10 \mathrm{mM}$ Tris- $\mathrm{HCl}, 50 \mathrm{mM} \mathrm{KCl}, 1.5 \mathrm{mM} \mathrm{MgCl}_{2}$, $200 \mathrm{uM}$ of each dNTP). The thermocycle programme was $5 \mathrm{~min}$ at $95^{\circ} \mathrm{C}, 30$ cycles of $30 \mathrm{~s}$ at $95^{\circ} \mathrm{C}, 30 \mathrm{~s}$ at $55^{\circ} \mathrm{C}, 90 \mathrm{~s}$ at $72^{\circ} \mathrm{C}$, then $5 \mathrm{~min}$ at $72^{\circ} \mathrm{C}$.

\section{Internal Transcribed Spacer (ITS)}

The Gyrodactylus salaris 18S rDNA forward primer ITS-1 5'-TTTCCGTAGGTGAACCT-3' correspond-

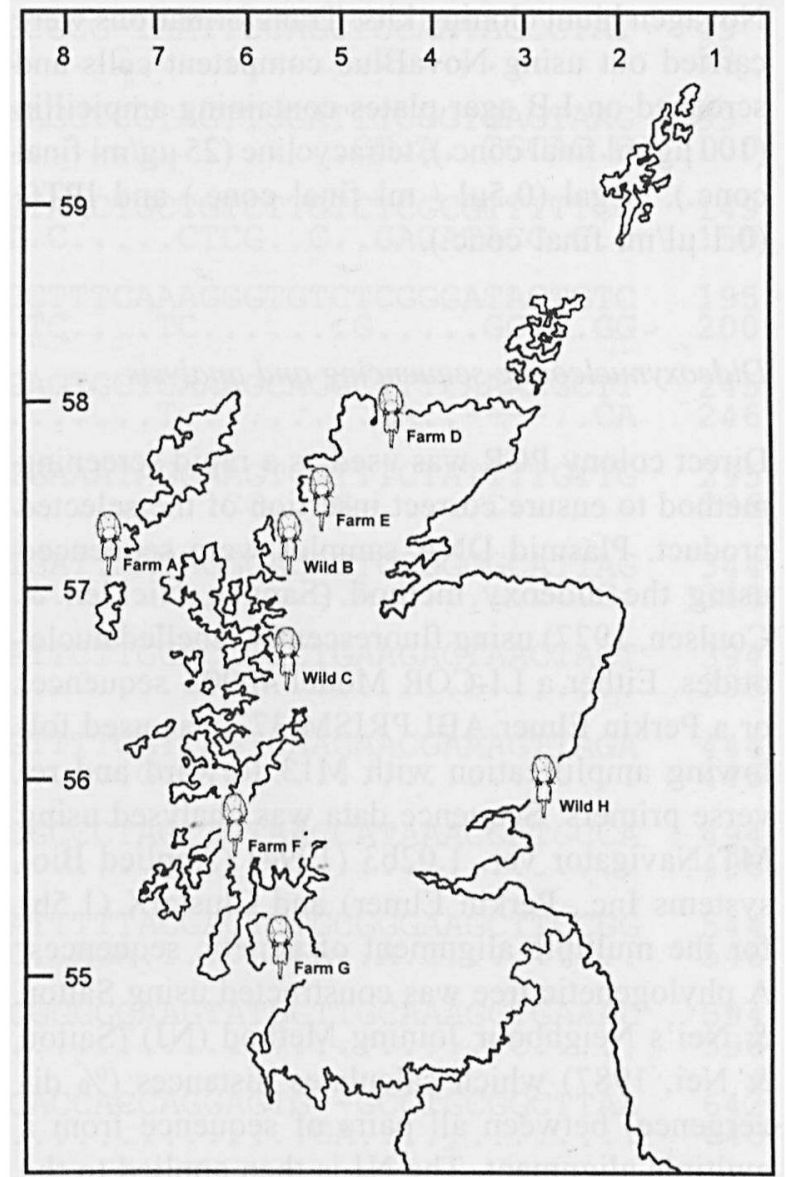

Fig. 1. Map of Lepeophtheirus salmonis collection sites in Scotland. The sequence of ITS- 1 was determined and compared for individual lice collected from sites A-D. Lice from these sites and from sites $\mathrm{E}-\mathrm{H}$ were used to determine a partial sequence of the 18S rRNA gene.

ing to the annealing position 1,938-1955 (Cunningham, McGillivray \& MacKenzie, 1995) and the Schistosoma 28S rDNA reverse primer ITS-2 5'-TCCTCCGCTTAGTGATA-3' (Littlewood \& Johnston, 1995) were used as primers in the PCR. The PCR reaction mix was as for the $18 \mathrm{~S}$ rDNA amplification. The thermocycling programme was $5 \mathrm{~min}$ at $95^{\circ} \mathrm{C}, 30$ cycles of $60 \mathrm{~s}$ at $95^{\circ} \mathrm{C}, 90 \mathrm{~s}$ at $52^{\circ} \mathrm{C}$, $120 \mathrm{~s}$ at $72^{\circ} \mathrm{C}$, then $5 \mathrm{~min}$ at $72^{\circ} \mathrm{C}$.

\section{Cloning into pMOS Blue T-vector}

PCR products were purified and ligated into $\mathrm{pMOS}$ Blue T-vector (pT7Blue) (Amersham, UK) using 
Novagen blunt cloning kits. Transformations were carried out using NovaBlue competent cells and screened on LB agar plates containing ampicillin $(100 \mu \mathrm{g} / \mathrm{ml}$ final conc.), tetracycline $(25 \mu \mathrm{g} / \mathrm{ml}$ final conc.), X-gal (0.5 $\mu \mathrm{l} / \mathrm{ml}$ final conc.) and IPTG $(0.1 \mu \mathrm{l} / \mathrm{ml}$ final conc.).

\section{Dideoxynucleotide sequencing and analysis}

Direct colony PCR was used as a rapid screening method to ensure correct insertion of the selected product. Plasmid DNA samples were sequenced using the dideoxy method (Sanger, Nicklen \& Coulsen, 1977) using fluorescently labelled nucleotides. Either a LI-COR Model 4000L sequencer or a Perkin Elmer ABI PRISM 377 was used following amplification with M13 forward and reverse primers. Sequence data was analysed using MT Navigator ver. 1.02b3 (1994) (Applied Biosystems Inc., Perkin Elmer) and ClustalX (1.5b) for the multiple alignment of genetic sequences. A phylogenetic tree was constructed using Saitou \& Nei's Neighbour Joining Method (NJ) (Saitou \& Nei, 1987) which calculates distances (\% divergence) between all pairs of sequence from a multiple alignment. The $\mathrm{NJ}$ is then applied to the distance matrix and the bootstrap tree which provides confidence estimates for the groupings was drawn using Treeview 1.5 (Page, 1996).

\section{Results}

\section{a) $18 S$ ribosomal DNA}

The precise sequence for a $0.9 \mathrm{~kb}$ fragment of the $18 \mathrm{~S}$ ribosomal RNA gene was determined from eight sea lice ( 2 from each site -2 wild and 2 farmed sites), using two clones per specimen (i.e. a total of 16 clones) and from both forward and reverse sequences for each clone. Further, the DNA for this region was extracted, purified, cloned and sequenced for lice taken from.the same samples on two separate occasions placing increased confidence in the sequence identity. A 904 nucleotide (nt) sequence was determined (Fig. 2) for each clone with no differences between clones, between indi- vidual lice or between sites. The most similar crustacean 18S previously recorded is that of the branchiuran parasite Argulus nobilis with a similarity of $83.92 \%$ (914 nt) (Abele, Kim \& Felgenhauer, 1989). The nucleotide sequence for this partial sequence of the $18 \mathrm{~S}$ ribosomal RNA gene has been submitted to the GenBank Data Library under the acquisition number AF043979.

\section{b) Internal Transcribed Spacer (ITS-1)}

A total of twenty sea lice from six sites ( 3 wild and $3 \mathrm{farm}$ ) were prepared for cloning. Two or three clones from each louse were prepared for sequencing. Forward and reverse sequences for each clone were obtained, aligned and the sequences compared. Where differences occurred in the forward and reverse sequence and / or between clones from each louse, the sequences were rejected. A total of thirteen lice from four sites ( 2 wild and 2 farm) were selected and compared and a 454 nucleotide sequence was derived (Fig. 3). Between all samples $(n=13)$ there was an overall $92.14 \%$ similarity in nucleotide sequences with 28 transitions, 28 transversions, 17 indels and 3 inversions (between 6 nucleotide positions). Within the ITS-1 sequence, there are three highly conserved regions between nucleotides 1-32, 91/94-183/189 ( $x / y$ denotes the range across all specimens), and $410 /$ $411-446 / 456$ and one hypervariable region between base pairs 289/299-401/411. The similarity between all sequences is given in Table 1 and a phylogenetic tree produced from determined bootstrap values (1000 iterations) is given in Fig. 4. To summarize, the percentage similarity between lice within farm sites was $\mathbf{9 9 . 7 1 \%}$ and $95.72 \%$ for sites $A$ and D respectively and $86.90 \%$ and $86.03 \%$ between lice from within wild sites B and C. This is clearly shown in Fig. 4 where lice from salmon held in farm cages cluster together in the dendrogram, whilst sea lice from wild salmonids are distributed throughout the dendrogram.

A sequence has been submitted to GenBank (acquisition no. AF043980 for a louse collected from a farmed salmonid (site D)). The most similar crustacean sequence in GenBank was the ITS sequence for the ostracod Darwinula brasiliensis 
L. salmonis

A. nobilis

GGGCAAGTCTGGTGCCAGCAGCCGCG-TAATTCCAGCTCCAATAGCGTAT

L. salmonis

A. nobilis

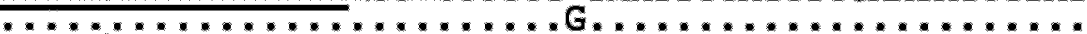

ATTAAAGTTGTTGCGGTCAAAAAGCTCGTAGTTGGATTTCGGTGAGTAAG

L. salmonis

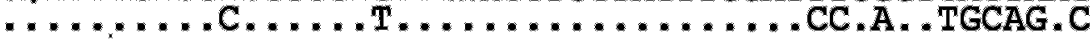

99

GAGGGGTTCATCTTGTTTGATGTAACTGCTGTCTTGTCTCGCGTTTTTCG

A. nobilis

.G.C. . . . GC. . CA.GGCGGT.C. . . . CTCG. .C. .GACATACC.C..

L. salmonis

ACAGAGGTTT---CGATGGTGCCTTTCAAAGGGTGTCTCGGGATACTGTC

GTT . . TC. . .GTC.CGC.AT . .TC. . . .TC. ...........GG.C.GG

A. nobilis

GGGTTTACTTTGAAAAAATTAGAGTGCTCAAAGCAGGCTTTTAAAAGCTT

L. salmonis

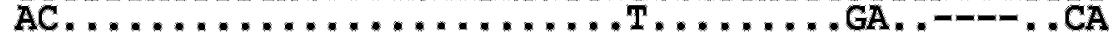

A. nobilis

GAATATTAGTGCATGGAATAATGAAATAGGAAGTCGTTTCTATTTTGTTG

L. salmonis

.....A.G...............................

A. nobilis

GTT-TTCGGAAATCGACTTAATGATTAATAGGGACAGTCGGGGGCATTAG

L. salmonis

... . . . .... . . .GG.......................

245

246

A. nobilis

TATTCAGACGACAGAGGTGAAATTCTTGGACCGTCTGAAGACTAACTACT

A. nabilis

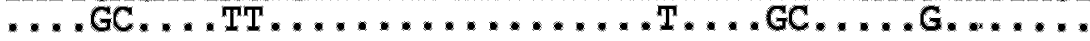

L. salmonis

GCGAAAGCATTTGCCAAGAATGTTTTCATTAATCAAGAACGAAAGTTAGA

A. nobilis

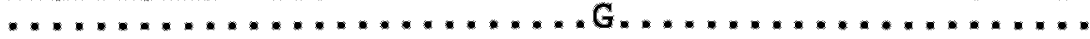

L. salmonis

GGTTCGAAGGCGATCAGATACCGCCCTAGTTCTAACCATAAACGATGCCA

A. nobilis

.................................

L. salmonis

GCTAGCGATCCGCAGTTGTTTATTTTTACGACTCTGCGGGAAGCTTCCGG

A.C. . . . . . . TC.GC . . AC. . AGA.T. . . . GA. . A.C. . . . . . .

346

A. nobilis

GAAACCAAAGCTTTTGGGTTCCGGGGGAGTATGGTTGCAAAGCTGAAAC

L. salmonis

........ . . . .......................

L. salmonis

TTAAAGGAATTGACGGAAGGGCACCACCAGGAGTG--GCCTGCGGCTTAA

A. nobilis

L. salmonis

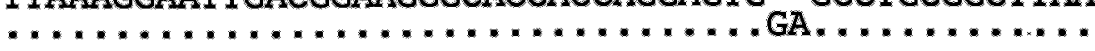

TTTGACTCAACACGGGAAATCTCACCAGGCCCGGACACTGGAAGGATTGA

A. nobilis

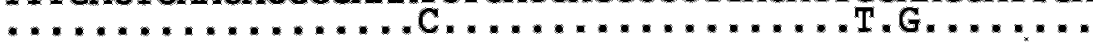

A. salmonis

CAGATTGAGAGCTCTTTCTCGATTCGGTGGGTGGTGGTGCATGGCCGTTC $\ldots \ldots \ldots \ldots \ldots \ldots \ldots$. . . . . . . . . . . . . . .

TTAGTTGGTGGAGTGATTTTCTGGTTAATTCCGATAACGAACGAGACTC

A. Salmonis

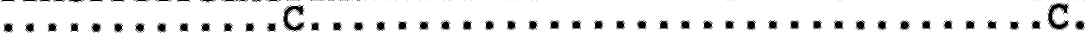

TGTCCTGCTAAATAGAGTTAACGTCATTTTTTTTGCGTTAACTTTTCTTC .T. . . . . . . . GCGG. .GA. . C.CA.GCAGTGTC....G......

A. salmonis

L. salmonis

A. nobilis

TTAGAGGGACTGGTGGCGTCTAGCCACACGAGATTGAGCAATAACAGGTC

L.: salmonis

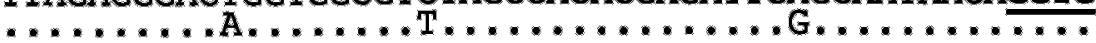

Fig. 2. The determined partial 18S ribosomal RNA gene sequence from Lepeophtheirus salmonis is given and compared to Argulus nobilis (Branchiurax Crustacea). Letters in bold and underlined represent the primers used for PCR amplification.

Pinto \& Kotzian (Schon, Butlin, Griffiths \& Martens, 1998) with a similarity of $47.94 \%$ (315 nt).

\section{Discussion}

The ability to identify different sea lice populations will make a major contribution to understand- ing characteristics including the dispersion and epidemiology of sea lice infections and contribute information to the ongoing dispute between salmon farming and wild salmonid fishery interests. To begin investigating the population characteristics of $L$. salmonis, two regions of the sea louse genome, namely $18 \mathrm{~S}$ rDNA and ITS-1 were targeted. No variation in a partial fragment (904 base pairs) 
Farm $A_{1}$
Farm $A_{2}$
Farm $A_{3}$
Farm $A_{4}$
Wild $B_{1}$
Wild $B_{2}$
Wild $C_{1}$
Wild $C_{2}$
Farm $D_{1}$
Farm $D_{2}$
Farm $D_{3}$
Farm $D_{4}$
Farm $D_{5}$

Farm $A_{1}$ Farm $A_{2}$ Farm $\mathrm{A}_{3}$ Farm $A_{4}$ Wild $B_{1}$ Wild $B_{2}$ Wild $\mathrm{C}_{1}$ Wild $c_{2}$ Farm $D_{1}$ Farm $D_{2}$ Farm $D_{3}$ Farm $D_{4}$ Farm $D_{5}$

Farm $A_{1}$ Farm $A_{2}$ Farm $\mathrm{A}_{3}$ Farm $A_{4}$ Wild $B_{1}$ Wild $\mathrm{B}_{2}$ Wild $\mathrm{C}_{1}$ Wild $\mathrm{C}_{2}$ Farm $D_{1}$ Farm $D_{2}$ Farm $D_{3}$ Farm $D_{4}$ Farm $D_{5}$
TTPCCGTAGGTGAACCTGCGGAAGGATCATTAATGCCAATTATACTACTAGCGTCCTC-TTTCCGTAGGTGAACCTGCGGAAGGATCATTAATGCCAATTATACTACTAGCGTCCTCTTTCCGIAGGTGAACCTGCGGAAGGATCATTAATGCCAATTATACTACTAGCGTCCTC-TTTCCGTAGGTEAACCTGCGGAAGGATCATTAATGCCAATTATACTACTAGCGTCCTC-TTTCCGTAGGTGAACCTGCGGAAGGATCATTAATGCCAATTATACTACTAGCGTCCTC-TITCCGTAGGTGAACCTGCGGAAGGATCATTAGTGCCAATTATTCTACTAGCGTCGTTTC TTCCGTAGGTGAACCTGCGGAAGGATCATTAATGCCAATTATACTACTAGCGTCCTC-TTTCCGIAGGTGAACCTGCGGAAGGATCATTAGTGCCAATTATTCTACTAGCGTCGTTTC TTCCGTAGGTGAACCTGCGGAAGGATCATTAATGCCAATTATACTACTAGCGTCCTC-TITCCGTAGGTGAACCTGCGGAAGGATCATTAGTGCCAATTATTCTACTAGCGTCGTTTC TTTCCGTAGGTGAACCTGCGGAAGGATCATTAGTGCCAATTATTCTACTAGCGTCGTTTC TTTCCGTAGGTGAACCTGCGGAAGGATCATTAGTGCCAATTATTCTACTAGCGTCGTTTC TTTCCGTAGGTGAACCTGCGGAAGGATCATTAGTGCCAATTATTCTACTAGCGTCGTTTC

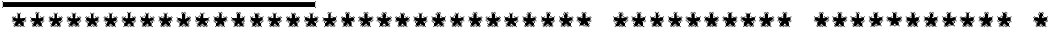

---ACGGGCGCTGTAAACTTTAAAAAAAAAAAAAAGAAAATTTTCAACGGTGGATATCT - - ACGGGCGCTGTAAACTTTAAAAAAAAAAAAAAGAAAATTTTCAACGGTGGATATCT ---ACGGGCGCTGTAAACTTTAAAAAAAAAAAAAAGAAAATTTTCAACGGTGGATATCT - - ACGGGCGCTGTAAACTTTAAAAAAAAAAAAAAGAAAATTTTCAACGGTGGATATCT - - ACGGGCGCTGTAAACTTTAAAAAAAAAAAAAAGAAAATTTTCAACGGTGGATATCT ACG-ACGG-CGCTGTAAACTTTAAAACAAAAAAAAAGAAAATTTTCAACGGTGGATATCT ---ACGGGCGCTGTAAACTTTAAAAAAAAAA----GAAAATTTTCAACGGTGGATATCT ACG-ACGG-CGCTGTAAACTTTAAAACAAAAAAAAAGAAAATTTTCAACGGTGGATATCT - --ACGGGCGCTGTAAACTTTAAAAAAAAAA--- GAAAATTTTCAACGGTGGATATCT ACGGACGG-CGCTGTAAACTTTAAAACAAAAAAAAAGAAAATTTTCAACGGTGGATATCT ACGGACGGGCGCTGTAAACTTTAAAACAAAAAAAAAGAAAATTTTCAACGGTGGATATCT ACG-ACGG-CGCTGTAAACTTTAAAACAAAAAAAAAGAAAATT'TCAACGGTGGATATCT ACG-ACGG-CGCTGTAAACTTTAAAACAAAAAAAAAGAAAATTTTCAACGGTGGATATCT $\star * * * * * * * * * * * * * * * * * * * * * * * * *$

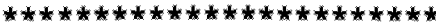

58

58

58

58

58

60

58

60

58

60

60

60

60

AGGTTCTCGTACCGAGGAAGAACGCAGCGAACTGCGATAAGCAATGCGAATTGCAGAACG AGGTTCTCGTACCGAGGAAGAACGCAGCGAACTGCGATAAGCAATGCGAATTGCAGAACG AGGTTCTCGTACCGAGGAAGAACGCAGCGAACTGCGATAAGCAATGCGAATTGCAGAACG AGGTTCTCGTACCGAGGAAGAACGCAGCGAACTGCGATAAGCAATGCGAATTGCAGAACG AGGTTCTCGTACCGAGGAAGAACGCAGCGAACTGCGATAAGCAATGCGAATTGCAGAACG AGGTTCTCGTACCGAGGAAGAACGCAGCGAACTGCGATAAGCAATGCGAATTGCAGAACG AGGTTCTCGTACCGAGGAAGAACGCAGCGAACTGCGATAAGCAATGCGAATTGCAGAACG AGGTTCTCGTACCGAGGAAGAACGCAGCGAACTGCGATAAGCAATGCGAATTGCAGAACG AGGTTCTCGTACCGAGGAAGAACGCAGCGAACTGCGATAAGCAATGCGAATTGCAGAACG AGGTTCTCGTACCGAGGAAGAACGGAGCGAACTGCGATAAGCAATGCGAATTGCAGAACG AGGTTCTCGTACCGAGGAAGAACGCAGCGAACTGCGATAAGCAATGCGAATTGCAGAACG AGGTTCTCGTACCGAGGAAGAACGCAGCGAACTGCGATAAGCAATGCGAATTGCAGAACG AGGTTCTCGTACCGAGGAAGAACGCAGCGAACTGCGATAAGCAATGCGAATTGCAGAACG

Fig. 3. The determined 454 nucleotide sequence for the internal transcribed spacer (ITS1) from different populations of Lepeophtheirus salmonis. Letters in bold and underlined represent the primers used for PCR amplification. Stars represent areas of identity between sequences.

of the 18S rRNA gene was found between samples of lice collected from four different sites. The $18 \mathrm{~S}$ rRNA gene was found to be highly conserved between samples demonstrating that the " $L$. salmonis" population from farmed and wild salmonids collected in Scottish waters represents a single species i.e. without cryptic species present.

Sequences of the intergenic spacer ITS-1 how- ever, were variable between sites with greater similarity demonstrated between individual lice taken from farmed salmonids than those taken from wild salmonids. Variation within the ITS-1 region is not uncommon and has been reported from Schistosoma where both size and sequence variation among copies of the ITS-1 repeat within individuals occurs (Van Herwerden et al., 1998). Similarly, 
Farm $A_{1}$

Farm $A_{2}$

Farm $A_{3}$

Farm $A_{4}$

Wild $\mathrm{B}_{1}$

Wild $B_{2}$

Wild $C_{1}$

Wild $\mathrm{C}_{2}$

Farm $D_{1}$

Farm $D_{2}$

Farm $D_{3}$

Farm $D_{4}$

Farm $D_{5}$

Farm $A_{1}$

Farm $A_{2}$

Farm $A_{3}$

Farm $A_{4}$

Wild $B_{1}$

Wild $\mathrm{B}_{2}$

Wild $\mathrm{C}_{1}$

Wild $\mathrm{C}_{2}$

Farm $D_{1}$

Farm $D_{2}$

Farm $D_{3}$

Farm $D_{4}$

Farm $D_{5}$

Farm $\mathbf{A}_{1}$

Farm $\mathrm{A}_{2}$

Farm $A_{3}$

Farm $A_{4}$

Wild $B_{1}$

Wild $\mathrm{B}_{2}$

Wild $\mathrm{C}_{1}$

Wild $\mathrm{C}_{2}$

Farm $D_{1}$

Farm $D_{2}$

Farm $D_{3}$

Farm $D_{4}$

Farm $D_{5}$

Farm $A_{1}$

Farm $A_{2}$

Earm $A_{3}$

Farm $A_{4}$

Wild $B_{1}$

Wild $\mathrm{B}_{2}$

Wild $\mathrm{C}_{1}$

Wild $\mathrm{C}_{2}$

Farm $D_{1}$

Farm $D_{2}$

Farm $D_{3}$

Farm $D_{4}$

Farm $D_{5}$

Fig. 3. Continued.
CGAGTCATTGGATTTTCGAACGCAACTGGCGGTCTGACGCAACAATCAGACCATGTGTGT CGAGTCATTGGATTTTCGAACGCAACTGGCGGTCTGACGCAACAATCAGACCATGTGTGT CGAGTCATTGGATTT'TCAACGCAACTGGCGGTCTGACGCAACAATCAGACCATGTGTGT CGAGTCATTGGATTTTCGAACGCAACTGGCGGTCTGACGCAACAATCAGACCATGTGTGT CGAGTCATTGGATTTTCGAACGCAACTGGCGGTCTGACGCAACAATCAGACCATGTGTGT CGAGTCATTTGATTTTCGAACGCATTCGGCGGTCCGACGTAGCAATCGGACCATGTGTGT CGAGTCATTGGATTTTCGAACGCAACTGGCGGTCTGACGCAACAATCAGACCATGTGTGT CGAGTCATTTGATTITCGAACGCATTCGGCGGTCCGACGTAGCAATCGGACCATGTGTGT CGAGTCATTTGATTTTCGAACGCAACCGGCGGTCCGACGTAGCAATCGGACCATGTGTGT CGAGTCATTTGATTTTCGAACGCAT'TCGGCGGTCCGACGTAGCAATCGGACCATGTGTGT CGAGTCATTTGATTTTCGAACGCATTCGGCGGTCCGACGTAGCAATCGGACCATGTGTGT CGAGTCATTTGATTTTCGAACGCATTCGGCGGTCCGACGTAGCAATCGGACCATGTGTGT CGAGTCATTTGATTTTCGAACGCATTCGGCGGTCCGACGTAGCAATCGGACCATGTGTGT

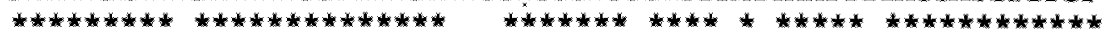

TTCAGTGTCAACAAAAATACCAATCTTAATGTGATTGAACTTCAGTTCTCTCATGAAATA TTCAGTGTCAACAAAAATACCAATCTTAATGTGATTGAACTTCAGTTCTCTCATGAAATA TTCAGTGTCAACAAAAATACCAATCTTAATGTGATTGAACTTCAGTTCTCTCATGAAATA TTCAGTGTCAACAAAAATACCAATCTTAATGTGATTGAACTTCAGTTCTCTCATGAAATA TTCAGTGTCAACAAAAATACCAATCTTAATGTGATTGAACTTCAGTTCTCTCATGAAATA TTCAGTGTCAACAAAACTCCCAATCTTAATGTGATTGAATTTCGATTCTCTCATGAAACA TTCAGTGTCAACAAAAATACCAATCTTAATGTGATTGAACTTCAGTTCTCTCATGAAATA TTCAGTGTCAACAAAACTCCCAATCTTAATGTGATTGAATTTCGATTCTCTCATGAAACA TTCAGTGTCAACAAAACTCCCAATCTTAATGTGATTGAATTCGATTCTCTCATGAAACA TTCAGTGTCAACAAAACTCCCAATCTTAATGTGATTGAATTTCGATTCTCTCATGAAACA TTCAGTGTCAACAAAACTCCCAATCTTAATGTGATTGAATTTCGATTCTCTCATGAAACA TTCAGTGTCAACAAAACTCCCAATCTTAATGTGATTGAATTTCGATTCTCTCATGAAACA TTCAGTGTCAACAAAACTCCCAATCTTAATGTGATTGAATTTCGATTCTCTCATGAAACA

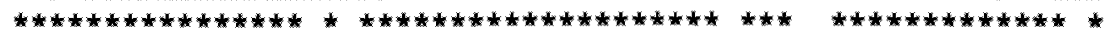

ACTGTGC-TTGCGGCTCGGACTGACGAAGTAGTCACCCGTCTCTGACGGCAGAGATTTCG ACTGTGC-TTGCGGCTCGGACTGACGAAGTAGTGACCCGTCTCTGACGGCAGTGATTTCG ACTGTGC-TTGCGGCTCGGACTGACGAAGTAGTCACCCGTCTCTGACGGCAGTGATTTCG ACTGTGC-TTGCGGCTCGGACTGACGAAGTAGTCACCCGTCTCTGACGGCAGTGATTTCG ACTGTGCCTTGCGGCTCGGACTGACGAAGTAGTCACCCGTCTCTGACGGCAGTGATTTCG AGAGGGGCTTGCGGCCTTCTCTGACGAAGTAGTCACCCGCTTGCGGA-_--TGATTTYCG ACTGTGCCTTGCGGCTCGGACTGACGAATTAGTCACCCGTCTCTGACGGCAGTGATTTCG AGAAGGGCTTGCGGCCTTCTCTGACGAATTAGTCACCCGCTTGCGGA----TGATTTCG AGAGGGGCTTGCGGCCTTCTCTGACGAAGTAGTCACCCGTCTCTGAA-----TGATTTCG AGAGGGGCTTGCGGCCTTCTCTGACGAAGTAGTCACCCGCTTGCGGA----TGATTTCG AGAGGGGCTTGCGGCCTTCTCTGACGAAGTAGTCACCCGCTTGCGGA-----TGATTTCG AGAGGGGCTTGCGGCCTTCTCTGACGAAGTAGTCACCCGCTIGCGGA----TGATTTCG AGAAGGGCTTGCGGCCTTCTCTGACGAATTAGTCACCCGCTTGCGGA-----TGATTTCG

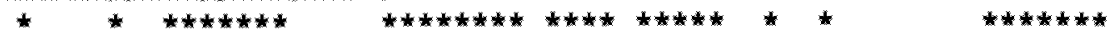

CCGGAAAGAGTTGAGAAGGTTCAGAGAAAACCTCTTTTGAGATCAAGACCTGAAATAGGC CCGGAAAGAGTTGAGAAGGTTCAGAGAAAACCTCTTTTGAGATCAAGACCTGAAATAGGC CCGGAAAGAGTTGAGAAGGTTCAGAGAAAACCTCTTTTGAGATCAAGACCTGAAATAGGC CCGGAAAGAGTTGAGAAGGTTCAGAGAAAACCTCTTTTGAGATCAAGACCTGAAATAGGC CCGGAAAGAGTTGAGAAGGTTCAGAGAAAACCTCTTTTGAGATCAAGACCTGAAATAGGC GCAGAGCTGGCTGAGAGGCTCCGGAGCAAACTTCTTTAACAATAACGACCTGAGACAGGC CCGGAAAGAATTGAGAAG-TTCAGAGAAAACCTCTTTTGAGATCAAGACCTGAAATAGGC GCAGAACTGGCTGAGAAGCTCCGGAGCAAACTTCTTTAACAATAACGACCTGAGACAGGC CCGGAAAGAGTTGAGAAGGTTCAGAGAAAACCTCTTTTGAGATCAAGACCTGAAATAGGC GCAGAGCTGGCTGAGAGGCTCCGGAGCAAACTTCTTTAACAATAACGACCTGAGACAGGC GCAGAGCTGGCTGAGAGGCTCCGGAGCAAACTTCTTTAACAATAACGACCTGAGACAGGC GCAGAGCTGGCTGAGAAGCTCCGGAGCAAACTTCTTTAACAATAACGACCTGAGACAGGC GCAGAACTGGCTGAGAAGCTCCGGAACAAACTTCTTTAACAATAACGACCTGAGACAGGC

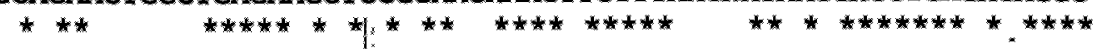

413

413

413

413

414

413

409

413

405

414

415

413

413 
Farm $A_{1}$
Farm $A_{2}$
Farm $A_{3}$
Farm $A_{4}$
Wild $B_{1}$
Wild $B_{2}$
Wild $C_{1}$
Wild $C_{2}$
Farm $D_{1}$
Farm $D_{2}$
Farm $D_{3}$
Farm $D_{4}$
Farm $D_{5}$

Fig. 3. Continued.

$\begin{array}{ll}\text { AAGATAACCCGCCCAACTTAAGCATATCACTAAGCGGAGGA } & 454 \\ \text { AAGATAACCCGCCAACTTAAGCATATCACTAAGCGGAGGA } & 454 \\ \text { AAGATAACCCGCCAACTTAAGCATATCACTAAGCGGAGGA } & 454 \\ \text { AAGATAACCCGCCCAACTTAAGCATATCACTAAGCGGAGGA } & 454 \\ \text { AAGATAACCCGCCCAACTTAAGCATATCACTAAGCGGAGGA } & 455 \\ \text { AAGATAACCCGCCAACTTAAGCATATCACTAAGCGGAGGA } & 454 \\ \text { AAGATAACCCGCCCAACTTAACCATATCACTAAGCGGAAGA } & 450 \\ \text { AAGATAACCCGCCCAACTTAAGCATATCACTAAGCGGAAGA } & 454 \\ \text { AAGATAACCCGCCAACTTAAGCATATCACTAAGCGGAGGA } & 446 \\ \text { AAGATAACCCGCCCAACTTAAGCATATCACTAAGCGGAGGA } & 455 \\ \text { AAGATAACCCGCCCAACTTAAGCATATCACTAAGCGGAGGA } & 456 \\ \text { AAGATAACCCGCCAACTTAAGCATATCACPAAGCGGAGA } & 454 \\ \text { AAGATAACCCGCCAACTTAAGCATATCACTAAGCGGAGA } & 454\end{array}$

454

454

454

454

454

450

454

455

456

454

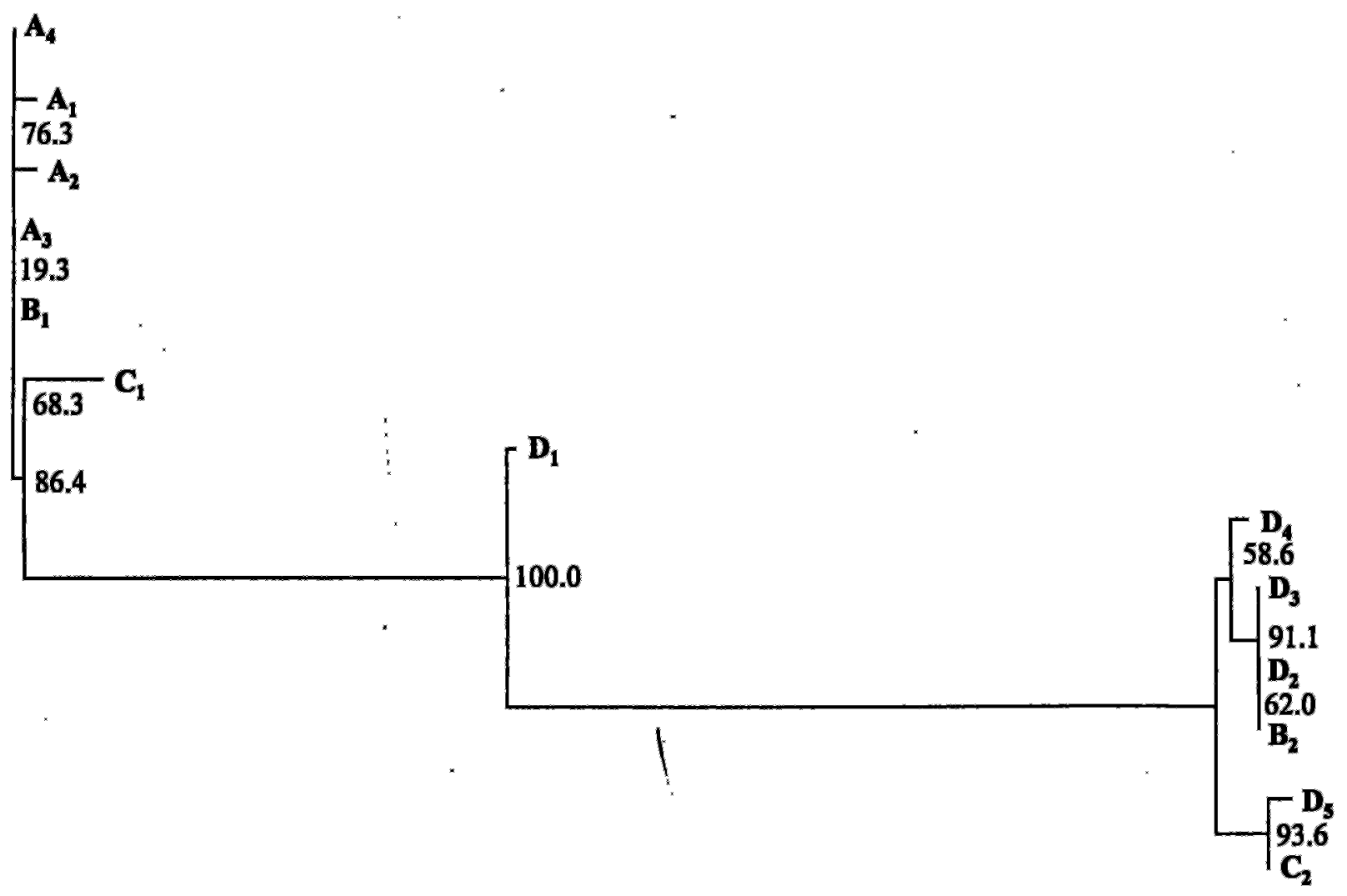

Fig. 4. Unrooted phylogenetic tree of Lepeophtheirus salmonis showing the relationship between the samples based on the ITS1 sequence constructed using Saitou \& Nei's Neighbour Joining Method (NJ). Samples $A_{1}-A_{4}$ and $D_{1}-D_{5}$ represent lice taken from two Atlantic salmon farms and samples $B_{1}-B_{2}$ and $C_{1}-C_{2}$ represent lice taken from two wild salmon collection sites. Nodes show bootstrap determined confidence estimates for each grouping expressed as percentages.

Diggles \& Adlard (1997) found 11 variable nucleotides in the 169-170 sequence representing ITS1 between 16 isolates of Cryptocaryon. Further, they reported genetic drift amounting to $2.9-3.5 \%$ divergence when the original samples of 3 isolates were compared to those maintained in a labora- tory over a 36 month period. The $0.29-4.28 \%$ divergence between samples of lice collected from farmed salmonids when compared to background levels (i.e. wild lice) of 13.10-13.97\% divergence between samples, may serve to illustrate that cage sites represent a bottleneck to genetic flow. A reduc- 
Table 1. Percentage similarity between nucleotide sequences for the internal transcribed spacer (ITS-1) obtained from four populations of Lepeophtheirus salmonis. Lice collected at sites A and D were from farmed salmonids whilst those lice collected from sites B and C were from wild salmonids.

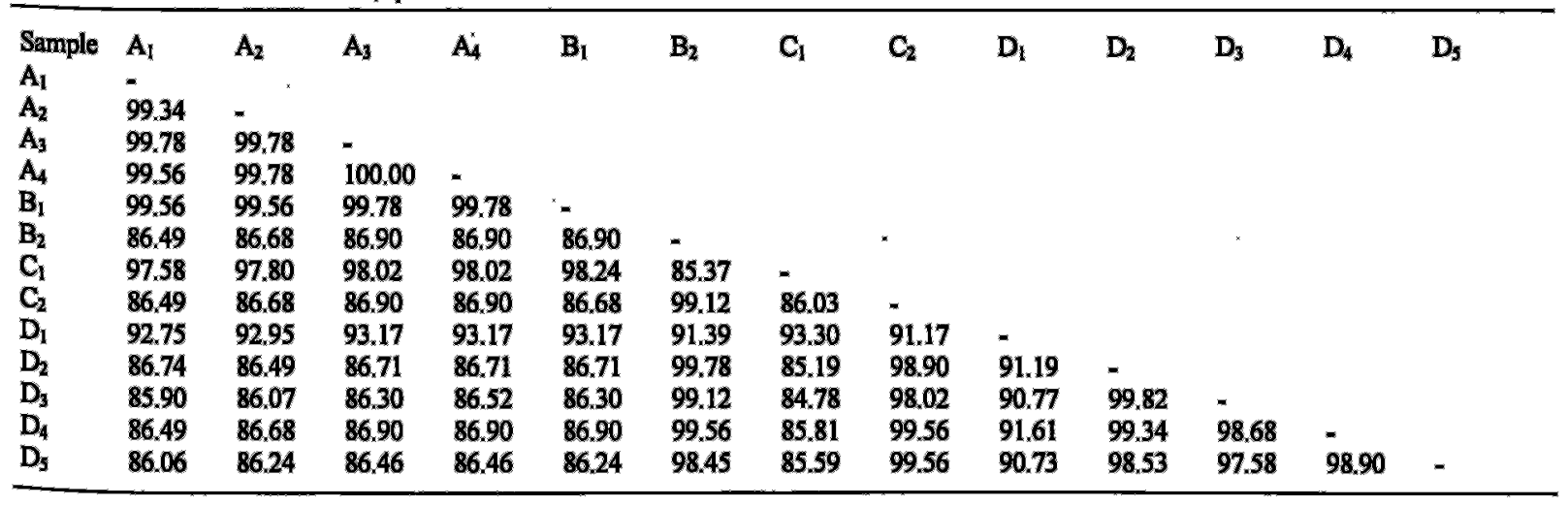

Summary statistics for lice within sites: within $A(n=6) 99.71 \pm 0.23 \%$ similarity; within $B(n=1) 86.90 \%$ similarity; within $C$ ( $n=$ 1) $86.03 \%$ similarity; and within $D(n=10) 95.72 \pm 4.04 \%$ similarity. Across all lice within farm sites $(n=36)$ there was $91.97 \pm$ $5.70 \%$ similarity; within wild sites $(n=6)$ there was $90.39 \pm 6.45 \%$ similarity: and across all lice and all sites $(n=78)$ there was 92.14 $\pm 5.88 \%$ similarity.

tion in the genetic variability or population isolation within farm sites, may stem from a combination of environmental and anthropogenic induced factors including farming intensity, loch flush rates, fallowing history, the retention of lice larval stages and the frequency of chemotherapeutant application. While fallowing is anticipated to eliminate the resident lice population, it is not known to what extent the gene pool is propagated across the fallowing period to the next stock of fish by either the interaction of the planktonic larvae with neighbouring farm sites or the activity of local salmonids, both of which may serve as a reservoir of genetic identity. Using the features of the hypervariable region identified within the ITS-1 region in this study, it may be possible to design population specific tags or primers that could subsequently be used to ascertain the origin of nauplii and copepodites on farmed and wild salmonids. The acquisition of such population markers could make significant inroads into our ability to monitor the dispersal, fate and impact of planktonic larvae not only on wild salmonids, but between farm sites and across fallow periods.

\section{Acknowledgements}

This work was supported by a BBSRC grant to $\mathrm{Dr}_{\mathrm{l}} \mathrm{C}$. Sommerville and Munbusho funding awarded to Professor T.

\section{References}

Abele LG, Kîm W, Felgenhauer BE. 1989. Molecular evídence for the inclusion of the phylum Pentastomida in the Crustacea. Mol. Biol. Evol. 66: 685-691.

Barta JR, Coles BA, Schito ML, Fernando M.A, Martin A, Danforth HD. 1998. Analysis of infraspecific variation among five strains of Eimeria maxima from North America. Int. J. Parasitol. 28: 485-492.

Blair D, Agatsuma T, Watonobe T, Okomoto M, Ito A. 1997. Geographical genetic structure within the human lung fluke, Paragonimus westermani, detected from DNA sequences. Parasitology 115: 411-417.

Chilton NB, Gasser RB, Beveridge I. 1995. Differences in a ribosomal DNA sequence of morphologically indistinguishable species within the Hypodontus macropi complex (Nematoda: Strongyloidea). Int. J. Parasitol. 25: 647-651.

Cunningham CO. 1997. Species variation within the internal transcribed spacer (ITS) region of Gyrodactylus (Monogenea: Gyrodactylidae) ribosomal RNA genes. J. ParasitoL. 83: 215219.

Cunningham CO, MeGillivray DM. MacKenzie K. 1995. Phylogenetic analysis of Gyrodactylus salaris Malmberg, 1957 based on the small subunit (18S) ribosomal RNA gene. Mol. Biochem. Parasitol. 71: 139-142.

De Meeüs T, Marin R, Renaud F. 1992. Genetic heterogeneity within populations of Lepeophtheirus europaensis (Copepoda: Caligidae) parasitic on two host species. Int. $J$. Parasitol. 22: 1179-1181.

-Diggles BK, Adlard RD. 1997. Intraspecific variation in Cryptocaryon irritans. J. Eukaryotic Microbiol. 44: 25-32.

Ellis RE, Sulston JE, Coulson AR. 1986. The rDNA of C. elegans: sequence and structure. Nucl. Acids Res. 14: 23452364. 
Hashimoto K, Watanobe T, Liu CX, Init I, Blair D, Ohnishi S,Agatsuma T. 1997. Mitochondrilal DNA and nuclear DNA indicate that the Japanese Fasciola species is $\mathbf{F}$ gigantica. Parasitol. Res. 83: 220-225.

Homan WL, Limper $L$, Verlaan M, Borst A, Vercammen $M$, Knapen F van. 1997. Comparison of the internal transcribed spacer, ITS-1, from Toxoplasma gondii isolates and Neospora caninum. Parasitol. Res. 83: 285-289.

Isdal E, Nylund A, Navdal G. 1997. Genetic differences among salmon lice (Lepeophtheirus salmonis) from six Norwegian coastal sites: evidence from allozymes. Bull. Eur. Assoc. Fish Pathol. 17: 17-22.

Littlewood DTJ, Johnston DA. 1995. Molecular phylogenetics of the four Schistosoma groups determined with partial $28 \mathrm{~S}$ ribosomal RNA gene sequences. Parasilology 111: 167-175.

Morgan JAT, Blaìr D. 1998. Relative merits of nuclear ribosomal internal transcribed spacers and mitochondrial $\mathrm{COI}$ and ND1 genes for dístinguishǐng among Echinostoma specíes (Trematoda), Parasitology 116: 289-297.

Nordhagen JR, Heuch PA, Schram TA. 1998. Size as an indicator of the origin of salmon lice Lepeophtheirus salmonis (Copepoda: Caligidae). Proceedings and Abstracts of the Fourth International Crustacean Congress. Amsterdam 2024 July, p.102.

Okamoto M,Agatsuma T, Kurosawa T, Ito A. 1997. Phylogenetic relationships of three hymenolepidid species inferred from nuclear ribosomal and mitochondrial DNA sequences. Parasilology 115: 661-666.

Page RDM. 1996. TREEVIEW: An application to display phylogenetic trees on personal computers. Comp. Applic. Biosciences 12 : 357-358.

Rinder H, Katzwinkel-Wladarsch S, Loscher T. 1997a. Evidence for the existence of genetically distinct strains of Enterocylozoon bieneusi. Parasitol. Res. 83: 670-672.

Rinder I, Rausch RL, Takahashi K, Kopp H, Thomschke A, Loscher T. 1997b. Limited range of genetic variation in Echinococcus multilocularis. J. Parasitol, 83: 1045-1050.

Romstad A, Gasser RB, Nansen P, Polerman AM, Chilton NB. 1998. Necator americanus (Nematoda: Ancylostomatidae) from Africa and Malaysia have different ITS-2 rDNA sequences. Int. J. Parasitol. 28: 61 I-615.

Saiki RK, Gelfand DH, Stofrel S, Scharf SJ, Higuchí R, Horn GT, Mullis KB, Erlich HA. 1988. Primer-directed enzymatic amplification of DNA with a thermostable DNA polymerase. Science 239: 487.

Sambrook J, Fritsch EF, Maniatis T. 1989. Molecular cloning: A laboratory manual. $2^{\text {nd }}$ edition. New York: Cold Harbor Laboratory Press.

Sanger F, Nicklen S, Coulson AR. 1977. DNA sequencing with chain terminating inhibitors. Proc. Nat. Ac. Sciences, USA 72: 5463-5467.

Schon I, Butlin RKB, Griffiths HI, Martens K. 1998. Slow molecular evolution in an ancient asexual ostracod. Proc. Roy. Soc. London, Ser. B 265: 235-242.

Shinn AP, Bron JE, Gray DJ, Sommerville C. (this volume) Elemental analysis of Scottish populations of the ectoparasitic copepod Lepeophtheirus salmonis (Krøyer, 1837). Contrib. Zool.

Shinn AP, Banks BA, Bron JE, Powell SF, Sommerville C. 1998. Differentiation of populations of the ectoparasitic copepod Lepeophtheirus salmonis (Krøyer, 1837) (Crustacea: Caligidae) in Scottish waters. British Soc. Parasitol, Spring Meeting, Abstracts, University of Exeter $5^{\text {th }}-8^{\text {th }}$ April, 102.

Stevenson LA, Gasser RB, Chilton NB. 1996. The ITS-2 rDNA of Teladorsagia circumcincta, $T$, trifurcata and $T$, davtiani (Nematoda: Trichostrongylidae) indicates that these taxa are one species. Int. J. Parasitol. 26: 1123-1126.

Todd CD, Walker AM, Wolff K, Northcott SJ, Walker AF, Ritchìe MG, Hoskins R, Abbott RJ, Hazon N. 1997. Genetic differentiation of populations of the copepod sea louse Lepeophtheirus salmonis (Krøyer) ectoparasitic on wild and farmed salmonids around the coasts of Scotland: Evidence from RAPD markers. J. Exp. Mar. Biol. Ecol. 210: 251-274.

Van Herwerden L, Blaîr D, Agatsuma T. 1998. Intra- and inter-specific variation in nuclear ribosomal internal transcribed spacer 1 of the Schistosoma japonicum species complex. Parasilology 116: 311-317.

Received: 4 January 1999 